\title{
Quotidiano de equipes de consultório na rua: tecendo redes para a promoção da saúde
}

The routine of the street outreach office team: weaving networks for health promotion El día a día de los equipos consultores de calle: tejiendo redes para la promoción de la salud

Selma Maria da Fonseca Viegas ${ }^{1}$ (1) Rosane Gonçalves Nitschke ${ }^{2}$ (D) Lucas Andreolli Bernardo ${ }^{3}$ (1)

Adriana Dutra Tholl ${ }^{2}$ (D)

Maria Aurora Rodriguez Borrego $0^{4,5}$ (]) Pablo Jesús López Soto 4,5 (1) Daniela Priscila Oliveira do Vale Tafner $^{6}$ (C)

1. Universidade Federal de São João del-Rei. Campus Centro-Oeste. Divinópolis, MG, Brasil.

2. Universidade Federal de Santa Catarina, Curso de Enfermagem. Florianópolis, SC, Brasil.

3. Prefeitura Municipal de Bombinhas. Bombinhas, SC, Brasil.

4. Universidad de Córdoba, Instituto Maimónides de Investigación Biomédica de Córdoba. Córdoba, España.

5. Hospital Universitário Reina Sofía de Córdoba. Córdoba, España.

6. Universidade Federal de Santa Catarina, Programa de Pós-Graduação em Enfermagem. Florianópolis, SC, Brasil.

Autora correspondente:

Selma Maria da Fonseca Viegas

E-mail: selmaviegas@ufsj.edu.br

Recebido em 24/06/2020.

Aprovado em 04/12/2020.

DOI:https://doi.org/10.1590/2177-9465-EAN-2020-0222

\section{Resumo}

Objetivo: compreender as potências e limites para a promoção da saúde de pessoas em situação de rua no quotidiano da equipe de Consultório na Rua. Método: estudo de casos múltiplos holístico-qualitativo, fundamentado na Sociologia Compreensiva do Quotidiano, realizado com dois informantes-chave e 20 profissionais de equipe de Consultório na Rua de duas capitais do Sul do Brasil. Utilizou-se a Análise de Conteúdo Temática. Resultados: as potências para a promoção da saúde de pessoas em situação de rua advêm da articulação da rede intrasetorial e intersetorial pela equipe de Consultório na Rua, além do vínculo e da redução de danos. Os limites enfrentados para desenvolver ações de promoção da saúde se deparam na especificidade e características desse público na terceirização da assistência social, na gestão, na política. Conclusões e implicações para a prática: a promoção da saúde ainda é um desafio para a equipe a ser superado perante as demandas de agravos, o tratamento do adoecimento e a lógica biomédica. As estratégias intrasetoriais e intersetoriais podem ser estabelecidas para alcançar ações em rede e efetivamente promover saúde, contemplando direitos fundamentais à vida de pessoas em situação de rua, mesmo em um quotidiano com condições desfavoráveis de viver e conviver.

Palavras-chave: Pessoas em Situação de Rua; Promoção da Saúde; Colaboração Intersetorial; Assistência à Saúde; Enfermagem.

\section{Abstract}

Objective: to understand the powers and limits for promoting the health of homeless people in the daily life of the Outreach office team. Method: a holistic-qualitative multiple case study, based on Comprehensive Sociology of Everyday Life, conducted with two key informants and 20 outreach office team professionals from two Southern Brazilian Capitals. We used the technique of thematic content analysis. Results: the powers for health promotion of homeless people come from the articulation of the intrasectoral and intersectoral network by the Outreach office team, in addition to bonding and harm reduction. The limits faced to develop health promotion actions are faced in the specificity and characteristics of this public, in the outsourcing of social assistance, in management, in politics. Conclusions and implications for practice: health promotion is still a challenge for the team to be overcome before demands of injuries, treatment of illness and biomedical logic. Intrasectoral and intersectora strategies can be established to achieve network actions and effectively promote health, contemplating fundamental rights to the lives of homeless people, even in a daily life with unfavorable conditions of living and living.

Keywords: Homeless Persons; Health Promotion; Intersectoral Collaboration; Delivery of Health Care; Nursing.

\section{Resumen}

Objetivo: comprender los poderes y límites para promover la salud de las personas que viven en las calles en la vida cotidiana del equipo de Consultório na Rua. Método: estudio de caso múltiple holístico-cualitativo, basado en la Sociología Comprensiva del Cotidiano, realizado con dos informantes clave y 20 profesionales del equipo de Consultório na Rua de dos capitales en el sur de Brasil. Se utilizó el análisis de contenido temático. Resultados: las competencias para promover la salud de las personas que viven en la calle provienen de la articulación de la red intrasectorial e intersectorial por parte del equipo Consultório na Rua, además del vínculo y la reducción de daños. Los límites que se enfrentan para desarrollar acciones de promoción de la salud se enfrentan en la especificidad y características de este público, en la externalización de la asistencia social, en la gestión, en la política. Conclusiones e implicaciones para la práctica: la promoción de la salud sigue siendo un desafío para el equipo a superar ante los problemas de salud, el tratamiento de la enfermedad y la lógica biomédica. Se pueden establecer estrategias intrasectoriales e intersectoriales para lograr acciones en red y promover eficazmente la salud, contemplando los derechos fundamentales a la vida de las personas que viven en la calle, incluso en una vida cotidiana con condiciones desfavorables para vivir y convivir.

Palabras clave: Personas Sin Hogar; Promoción de la Salud; Colaboración Intersectorial; Prestación de Atención de Salud; Enfermería 


\section{INTRODUÇÃO}

A saúde é o maior recurso para o desenvolvimento social, econômico e pessoal, assim como uma importante dimensão da qualidade de vida, precisando "ser vista como um recurso para a vida, e não como objetivo de viver". ${ }^{1: 1}$ Entretanto, ainda há populações em situação de extrema vulnerabilidade, como a população em situação de rua, para a qual persistem barreiras ao acesso e ao cuidado em saúde, mantendo-a invisível para o sistema público de saúde. ${ }^{2}$ Esse segmento populacional, vulnerado pela estrutura de vida cotidiana na rua, tem direito à cidadania e à atenção à saúde, e a equipe de Consultório na Rua $(\mathrm{eCR})$ se torna referência para o atendimento às necessidades de vida e saúde dessas pessoas. A vida na rua expõe quem vive nela a riscos que exigem da eCR uma abordagem específica. ${ }^{3}$

Essa equipe, em seu quotidiano, faz acontecer a assistência, o cuidado e a promoção da saúde às pessoas em situação de rua, em uma prática itinerante que perpassa a rede de saúde intersetorial e de solidariedade "mesclando-se às mesmas, não raras vezes, sob a tensão, buscando articulação para o atendimento daqueles que, até então, encontravam-se invisíveis nos/aos cenários do Sistema Único de Saúde (SUS) no Brasil". 4:253 Estar junto à população de rua em diferentes cenários faz dessa equipe presença no real, identificando e priorizando no quotidiano os diversos problemas e necessidades de saúde para ações em rede.

Assim, a eCR realiza suas atividades in loco, desenvolvendo ações compartilhadas e integradas às unidades de Atenção Primária à Saúde (APS), e, quando necessário, também com as equipes dos Centros de Atenção Psicossocial, dos serviços de Urgência e Emergência e de outros pontos de atenção da rede, ${ }^{5}$ garantido direito e acesso às pessoas em situação de rua.

O direito à saúde prevê o desfrute de saúde física e mental por todas as pessoas na sua integralidade e necessidade, implicando a promoção da saúde pela articulação intrasetorial e colaboração intersetorial por meio da formação da Rede de Atenção à Saúde (RAS), buscando integrar ações com as demais redes de proteção social, com ampla participação e controle social, conforme proposto na Política Nacional de Promoção da Saúde no Brasil. ${ }^{6}$

A promoção da saúde objetiva a equidade e a melhoria das condições e modos de viver, ampliando a potencialidade da saúde individual e coletiva, reduzindo vulnerabilidades e riscos à saúde decorrentes dos determinantes sociais, econômicos, políticos, culturais e ambientais. A promoção e defesa da saúde e da vida propõe reduzir as desigualdades sistemáticas, injustas e evitáveis, com respeito às diferenças em todas as dimensões para o direito universal à saúde. ${ }^{6}$

Ao tratar o direito à saúde, na perspectiva de direitos humanos, seu pleno exercício depende também do direito à vida, à liberdade, à educação, à participação política, à moradia adequada, à alimentação, entre outros. No entanto, a garantia desses direitos não se dá de forma equitativa para todas as pessoas, como para as pessoas em situação de rua, o que pode produzir situações de iniquidades e violações de direitos. As estratégias para a redução das iniquidades na garantia do direito à saúde como direito humano fundamental fazem parte dos princípios e valores de universalidade, acessibilidade, integralidade, qualidade e inclusão em saúde de uma maneira coesa e integrada, de forma a alcançar a promoção da saúde. Quando os direitos humanos são negados, prejudicam-nos enquanto indivíduos e a sociedade como um todo, que se desenvolverá menos e terá níveis de segurança e de bem-estar menores.?

Como campo emergente, a inclusão em saúde precisa fornecer serviços sociais e de saúde integrados para a sobreposição de grupos marginalizados. A capacidade de ter políticas de saúde e social, para responder às necessidades das populações marginalizadas, é um indicador-chave de qualidade. A extrema desigualdade em saúde que persiste em países de alta renda é evidenciada em um estudo de revisão sistemática, destacandose como resolubilidade que a política de saúde de inclusão deve se basear nas evidências que desencadeiam a exclusão para avultar os benefícios sociais e econômicos e evitar a exclusão social devido à pobreza, marginalização e multimorbidade. ${ }^{8}$

Estudos que tiveram como análise a saúde das pessoas que fazem da rua a sua moradia apresentam, em sua maioria, o perfil epidemiológico, comorbidades, incluindo as doenças mentais e uso de drogas lícitas e ilícitas, o maior risco de doenças transmissíveis devido a vários fatores e exposições aumentadas como a falta de acesso à higiene e saúde. Apontam os avanços das políticas públicas específicas e os desafios de garantir a atenção integral com estratégias efetivas de cuidado para responder às necessidades básicas, demandas de saúde e dignidade dessas pessoas. . $^{9-12}$

Dentre os pontos da RAS, o uso de serviços de emergência e as internações hospitalare ${ }^{13,14}$ foi mais significativo entre as pessoas em situação de rua com comorbidades e uso de substâncias. ${ }^{15,16} \mathrm{~A}$ frequência de atendimento à emergência $e$ hospitalizações, às vezes, pode ser evitada quando as pessoas em situação de rua recebem cuidados primários de alta qualidade, orientações, benefícios sociais e de serviços de apoio, de longa duração, para respostas às necessidades básicas, reduzindo assim gastos com intervenções de saúde evitáveis. ${ }^{13}$

Outros estudos avançam nas ações promocionais da saúde ao trabalharem com aconselhamento para cessação do tabagismo e farmacologia para fumantes,${ }^{17}$ redução de danos, ${ }^{18}$ intervenção interdisciplinar após alta hospitalar, ${ }^{19}$ práticas de atividade física com melhorias na saúde mental, social e reinserção na comunidade. ${ }^{20}$ No entanto, muito ainda precisa ser feito para garantir a saúde e sua promoção, para qualidade de vida das pessoas, mesmo em situação de rua, ${ }^{10,20}$ o que justifica a relevância desta temática.

Para oferecer oportunidades de acesso a bens e serviços a todos, é fundamental a promoção da cidadania e o acesso aos direitos humanos básicos. Em vista disso, precisa-se do reconhecimento das necessidades das pessoas em situação de rua e promoção de ações corresponsabilizadas em rede intrasetoriais e intersetoriais que busquem respondê-las. Há, portanto, o imperativo de todos os envolvidos, em especial a 
gestão, implicarem-se com o conjunto de elementos que determina a oferta das ações assistenciais, de cuidado e promoção da saúde na organização e na gestão de serviços e equipes. ${ }^{21}$

Diante desse contexto questiona-se: como os profissionais da eCR planejam e desenvolvem ações de promoção da saúde considerando as singularidades e necessidades das pessoas em situações de rua? Como se contextualizam, no quotidiano, as potências para a promoção da saúde das pessoas em situações de rua? Como são vivenciados os limites para a promoção da saúde das pessoas em situações de rua?

Este estudo teve por objetivo compreender as potências e limites para a promoção da saúde das pessoas em situação de rua no quotidiano da eCR.

Entendendo-se o quotidiano como "a maneira de viver dos seres humanos que se mostra no dia a dia, expresso por suas interações, crenças, valores, símbolos, significados, imagens e imaginário. Delineando assim, seu processo de viver, em um movimento de ser saudável e adoecer, pontuando seu ciclo vital". 22:8

\section{MÉTODO}

Trata-se de um estudo de casos múltiplos holístico-qualitativo, ${ }^{23}$ fundamentado na Sociologia Compreensiva do Quotidiano de Michel Maffesoli. ${ }^{24}$

Este estudo contém dois casos, definidos pelos cenários, que são duas capitais do Sul do Brasil. A delimitação desses casos múltiplos se fez pela análise de cada caso individualmente, sob uma unidade única de análise que se relaciona às questões e proposição da pesquisa "A promoção da saúde das pessoas em situação de rua no quotidiano da equipe de Consultório na Rua"; constitui-se, portanto, um estudo de casos múltiplos holístico-qualitativo e pela definição da coleta de dados em todas as eCR existentes nos municípios, entrevistando os profissionais até a saturação dos dados pela replicação literal. A lógica da replicação literal prevê resultados semelhantes, não da amostragem, ou seja, após revelar evidências significativas por meio do primeiro caso, o objetivo imediato deste estudo foi replicar essas evidências, conduzindo o segundo caso. ${ }^{23}$

A Sociologia Compreensiva do Quotidiano propõe a compreensão dos fenômenos sociais, alvitrando uma razão sensível, que busca valorizar os saberes do quotidiano e o senso comum. Portanto, torna-se oportuno lançar esse olhar sobre o objeto de estudo, já que se referencia a tudo o que diz respeito à vida quotidiana, às experiências vividas, às crenças e às ações dos sujeitos nos seus ambientes de relações, ${ }^{24,25} \mathrm{em}$ casos/cenários específicos da vivência cotidiana e real em eCR, para ser uma manifestação concreta da abstração do fenômeno contemporâneo estudado. ${ }^{23}$

Os participantes da pesquisa foram 20 profissionais de cinco eCR e 02 informantes-chave, gestores nacional/municipal, cujas participações foram voluntárias. Incluíram-se no estudo profissionais que atuam nas eCR das duas capitais do Sul do Brasil por um período mínimo de seis meses. A participação dos informantes-chave se justifica por terem contribuído com informações precisas sobre o objeto pesquisado e foram indicados pelos participantes deste estudo. Excluíram-se profissionais em férias ou afastados do trabalho no período da coleta de dados. Quanto às pessoas que não atenderam aos critérios de inclusão, somente uma tinha menos de seis meses de atuação na eCR. Apenas $3(13,6 \%)$ recusaram participar da pesquisa. A coleta de dados foi encerrada no momento em que se constatou a saturação dos dados, isto é, quando se obteve um número suficiente de infomações replicadas, configurando a replicação literal por resultados semelhantes nos dois $\operatorname{casos}^{23}$ sem desprezar as informações novas e significativas para este estudo.

Como fontes de evidências dos dados, utilizaram-se a entrevista individual aberta intensiva, com roteiro semiestruturado, e registros em notas de campo. A entrevista foi realizada pela pesquisadora, gravada por meio digital, transcrita na íntegra e validada pela leitura do participante. O tempo médio das entrevistas foi de 39 minutos e nove segundos. As notas de campo foram utilizadas com fins operacionais: direcionadas para os procedimentos operacionais da pesquisa, descrevendo características dos municípios e das eCR; a organização do trabalho da eCR; a logística; os pontos de apoio nas instituições de referência e na RAS, além dos fatos relevantes da coleta de dados.

A análise dos dados foi realizada por meio da Análise de Conteúdo Temática, ${ }^{26}$ obedecendo à técnica analítica da síntese cruzada dos casos. ${ }^{23}$ Foi definida pelo critério semântico, isto é, pela análise dos "significados" segundo pré-análise, exploração do material, tratamento dos resultados, inferência e a interpretação, ${ }^{26}$ considerando o referencial teórico da Sociologia Compreensiva e do Quotidiano. ${ }^{24}$ Este artigo apresenta a categoria "Consultório na Rua e rede de atenção na promoção da saúde das pessoas em situação de rua: potências e limites no quotidiano".

Atenderam-se aos preceitos éticos que envolvem estudos com seres humanos. Os participantes assinaram o Termo de Consentimento Livre e Esclarecido. Para manutenção do sigilo das informações e anonimato dos participantes da pesquisa, foram utilizados códigos alfanuméricos na descrição dos resultados. Este estudo foi aprovado sob o Parecer 2.766.046 (Instituição Proponente) e sob o Parecer 2.837.324 (Instituição Co-Participante).

\section{RESULTADOS}

Dos 22 participantes deste estudo, 18 (81,8\%) são do sexo feminino. A idade variou de 28 a 57 anos, sendo a média de 43,3 anos. Quanto à escolaridade, seis têm formação técnica de nível médio, 16 têm nível superior e 11 são pós-graduados, dentre os quais 02 têm especialização em Consultório na Rua. $\mathrm{O}$ tempo médio de atuação na eCR foi de quatro anos e oito meses.

Os resultados, apresentados em tópicos, consideram as subcategorias de análise da categoria temática "Consultório na Rua e rede de atenção na promoção da saúde das pessoas em situação de rua: potências e limites no quotidiano".

A promoção da saúde é referida como um desafio a ser superado no quotidiano da eCR frente às demandas de agravos, ao tratamento do adoecimento e à lógica biomédica. Os resultados 
apresentam como são ou não planejadas e desenvolvidas as ações para efetivar a promoção da saúde, enfocando as potências e limites no quotidiano. Apontam a necessidade de reestruturação dos serviços e articulação com os demais componentes da rede de atenção para avançar na promoção de vida com mais qualidade para as pessoas em situação de rua, superando a exclusão institucional ainda vivenciada pelas características estigmatizantes que experimentam essas pessoas (Notas de Campo).

\section{Promoção da saúde das pessoas em situação de rua: como a eCR planeja e desenvolve as ações}

As ações de saúde realizadas no quotidiano das eCR buscam identificar as demandas das pessoas em situação de rua em uma abordagem integral e articulada à rede básica de saúde e à rede intersetorial (Notas de Campo).

A busca por superar as dificuldades para uma dimensão ampliada da atenção à saúde, que inclua os determinantes sociais desse viver e adoecer no espaço e tempo da rua, com vistas à promoção da saúde das pessoas em situação de rua, fez-se presente desde a implantação das eCR:

Desde o início, sempre tentamos ver quais são as necessidades dessas pessoas frente aos serviços que já existiam. O Consultório na Rua tem seis anos no município, mas a Abordagem Social tem 20, o Centro POP (Centro de Referência Especializado para População em Situação de Rua) já existe há 15 anos. Então, são serviços que já conheciam e podiam trazer quais necessidades que aquela população tinha. Claro que, no primeiro momento da criação do Consultório na Rua, teve uma quantidade de pacientes agravados pela falta de atendimento que essa população tinha, mas a gente não quis ficar só na parte curativa $\left(\mathrm{E}_{4}\right)$.

As oportunidades reduzidas para ações de promoção à saúde e como a eCR (não)planeja e (não)desenvolve essas ações no quotidiano foram relatadas:

Já tem um processo de trabalho muito consolidado, então não param para planejar ações de promoção da saúde. Eu tenho feito isso e tentando trazê-las mais pela via da prática mesmo. Não é só ficar "ah, vamos fazer uma educação permanente e conversar", não é só pela via do discurso que eu vou conseguir mudar, mas mostrar como fazer $\left(\mathrm{E}_{2}\right)$.

Eu, como médica, muito pouco! Mas eu corro atrás do prejuízo do que trabalho com a promoção da saúde. Do meu trabalho específico, vejo que estou sempre numa demanda de tratamento, tratamento... quem tem conseguido, e acredito, é a nossa psicóloga, que tem trazido essa possibilidade desse olhar de promoção. [...] como equipe, ainda está muito na demanda da doença. $A$ promoção está mais no individual, no quanto é possível trabalhar a redução de danos $\left(\mathrm{E}_{6}\right)$.

Estamos mais no tratamento do que na promoção. Faz muito teste rápido e tem bastante paciente não reagente, fazemos a orientação, mas tu não consegues acompanhar essas pessoas. [...] pega o paciente já acometido do agravo e trata o agravo $\left(\mathrm{E}_{3}\right)$.

\section{A rua como espaço de moradia e de sustento das pessoas em situação de rua: distribuições desiguais das determinações da saúde}

O promover a saúde perpassa pelas Organizações Não Governamentais (ONG) e entidades, o que leva a eCR a refletir sobre a rua como espaço de moradia e de sustento das pessoas em situação de rua e sobre a ação solidária de ajudar no combate à fome desses seres humanos perante a distribuição desigual das determinações da saúde:

Quando eu era pequena, batia assim na porta e pediase pão velho. A fome não é problema! Tem comida de montão! Se sair à noite, tem gente dormindo na praça com três marmitas, as ONG e as igrejas deixam. A pena que move as pessoas de quererem ajudar é muito pela comida. Então, teria que ter um projeto que as pessoas levassem suas doações para uma central [...] teria que ter uma reforma, muito se passa pela consciência cristã de querer ajudar. Com esse tipo de ajuda, as pessoas permanecem mais ainda na rua e falando mal do abrigo. Porque, é claro, na rua ela tem liberdade, no abrigo ela tem regras $\left(\mathrm{E}_{7}\right)$.

A moradia, a dependência química e a dor são abordagens singulares e de direito em um quotidiano de porta sempre aberta à pessoa em situação de rua:

Abordo questão de moradia, por que a pessoa está aqui? Tem algum vício? Gostaria de interromper ou de reduzir? Quer acompanhar conosco? Tento já abranger tudo, se a pessoa der essa liberdade também, porque tem muita gente que chega "Eu estou com dor, eu quero isso, outro dia eu volto e vejo se eu quero", e a gente tenta manter a porta sempre aberta $\left(\mathrm{E}_{5}\right)$.

\section{Potências na promoção da saúde de pessoas em situação de rua}

As potências para a promoção da saúde de pessoas em situação de rua advêm da articulação com a rede intrasetorial e intersetorial, realizada pelos profissionais da eCR. As ações em rede são dirigidas a indivíduos com agravo(s) ou de enfrentamento de determinantes sociais que vão além das demandas prioritárias dessas pessoas: 
Uma potência é a articulação em rede, porque não se faz promoção da saúde sozinho. Por mais que tenha saúde no nome, mas não é só da área da saúde. Principalmente pensando saúde no conceito ampliado, saindo dessa área clínica biológica. Se saúde é ter emprego, se saúde é ter casa, se saúde é acessar serviços públicos, eu tenho que acessar a rede. Então, para mim, é a articulação de rede, seria assim a máxima! Porque pensando nas dificuldades, nos limites institucionais, na precariedade. [...] então, a rede é a principal potência que a gente tem nas mãos, tanto pelo próprio efeito da união, da qualificação, mas porque a gente se une pelas dificuldades também. Une-se na precariedade, se une pela necessidade $\left(\mathrm{E}_{2}\right)$.

A organização da atenção à saúde no cenário da rua é vista como uma potência para a eCR por conhecer a realidade das pessoas em situação de rua, in loco, e construir o vínculo:

É difícil falar de potências para a promoção da saúde. Mas, o fato de estar mais na rua, acaba conseguindo conversar com as pessoas e ter uma convivência por um período maior. O vínculo de estar no lugar mais próximo de onde ele fica $\left(E_{6}\right)$.

Ações singulares, ao trabalhar a redução de danos no contexto da rua, configuram a promoção da saúde considerando a subjetividade, a condição e o meio social, proporcionando a conexão entre o cuidado de saúde e a ampliação da qualidade de vida pela redução de danos:

A prevenção primária? Estamos com uma população que em várias questões já é terciário. Pensando na promoção da saúde, a gente trabalha primeiro a questão da dependência química na visão de redução de danos $\left(\mathrm{E}_{14}\right)$.

Já jogamos futebol... roda de música, roda de conversa. Tudo isso reduz danos, por não estarem usando droga, então promove saúde $\left(\mathrm{E}_{16}\right)$.

A atenção à saúde e o cuidado junto às pessoas em situação de rua são considerados de alcance coletivo, uma vez que as ações e serviços passam a ser de relevância para a saúde pública, ao pensar essas condições do viver nas ruas como importante elemento dentro da relação dinâmica do processo saúde-doença:

Um dos maiores potenciais é trabalhar promoção da saúde. Muita gente não entende que trabalhar a saúde da população de rua é um trabalho para toda a sociedade, ainda acha que é muito pontual, que é para aquela pessoa em si, e não é. A gente sabe que é algo muito maior $\left(\mathrm{E}_{4}\right)$.

Dos 22 participantes, 10 apresentaram a noção preventiva e educativa ao abordarem o seu significado em relação à promoção da saúde das pessoas em situação de rua, evidenciando como a prevenção de riscos e agravos se configura nas ações e atividades cotidianas das eCR (Notas de Campo).

\section{Limites na promoção da saúde das pessoas em situação de rua}

Os limites enfrentados para desenvolver ações de promoção da saúde das pessoas em situação de rua se deparam na especificidade e características desse público, na terceirização da assistência social, na gestão, na política:

Foram vários baldes de água fria ao longo desses seis anos, porque trabalhar com promoção da saúde em si já é muito difícil! Para a população de rua, então, é quase impossível: para quê? Para essa população? Para que dispensar um trabalhador para fazer promoção da saúde para um tipo de população que não está nem procurando o serviço de saúde? [...] os limites são os entraves políticos que ainda acontecem, não é só para a população em situação de rua, acho que é uma questão mesmo política no País, a gente está em um retrocesso também. Mas, continuo achando que é mais difícil no que diz respeito à população em situação de rua $\left(\mathrm{E}_{4}\right)$.

Os limites de conhecimento, político, institucionais, todos, de toda ordem. [...] queixam muito dos limites institucionais das condições de trabalho. Por exemplo, faz muita falta um celular para estar na rua. O horário de funcionamento do Consultório é i-na-cei-tá-vel! [...] a rua tem rotina, a rua tem hora! De manhã eles dormem ou têm que passar a manhã no centro POP, porque só podem almoçar se estiverem lá dentro. Aíquem não está no horário do almoço no POP, o almoço deles é às duase meia, porque é depois das duas em diante que eles vão manguear os restaurantes, o que sobrou do almoço. Das três as 20 eu diria, porque canta bem e aí, às 20 horas escureceu, ali já é outra rotina, já é o uso $\left(\mathrm{E}_{2}\right)$.

A dificuldade é essa desestruturação da assistência social sendo terceirizada, e com comunicação difícil. [...] a assistência social lida com uma carência maior do que a saúde, de recurso, de profissionais. O fato de não conseguirmos trabalhar tanto juntos, diminui o potencial de fazer promoção. [...] outra questão é a equipe não ter outros profissionais, o educador social, o agente de saúde, assim acaba preso no biológico, no técnico $\left(\mathrm{E}_{6}\right)$.

Realmente é difícil trabalhar com a promoção da saúde com a população de rua sem ser a Atenção Básica, primária, emergencial $\left(\mathrm{E}_{7}\right)$.

Há a menção de que a promoção da saúde é de difícil articulação no quotidiano dos serviços, sendo interdependente da rede, da gestão e da política:

Se iremos conseguir fazer mais promoção da saúde, depende se a gestão é mais sensibilizada para atendimento dessa população. Se não é tão sensibilizada, ela vai manter a 
equipe fazendo o básico. $E$ aí, se depara com questões políticas, tínhamos um projeto que envolvia a cidadania das pessoas em situação de rua. [...] e que nos é cortado por uma questão política. [...] a população de rua ainda é muito foco deste tipo de politicagem, dificultando esse trabalho de promoção. Mas, o mais importante é ver no perfil de profissionais, o desejo de trabalhar com a promoção [...] fazer de uma forma grande, ampla, ainda é muito difícil, e quando a gente se evidencia isso acaba querendo ser tapado $\left(\mathrm{E}_{4}\right)$.

Considerando a análise múltipla dos casos, em um dos casos, $66,7 \%$ dos participantes abordaram a compreensão da promoção da saúde e de como as ações são ou não planejadas e desenvolvidas no quotidiano da eCR, considerando as condições básicas e suplementares do viver e conviver das pessoas em situação de rua. No outro caso, a promoção da saúde surgiu nas descrições de $14,3 \%$ dos participantes.

No entanto, pelo olhar observador em campo de pesquisa e pela convivência cotidiana com as eCR para a coleta de dados em ambas as realidades, as ações de promoção da saúde se mostram nesse quotidiano enquanto cena e cenário. Foram vistas ao presenciar momentos em que as necessidades cotidianas de cada uma das pessoas que procuraram atenção pela eCR, ou que foi abordada na amplitude do viver na rua, voltaram-se para a dimensão do ser e dos determinantes do viver em situação de rua. A fome, o frio, o vestuário, a falta de higiene corporal, o sono na rua, o uso abusivo de álcool e outras drogas, a violência, o adoecimento e a condição psicossocial foram revertidos por olhares humanizadores e em ações articuladas em rede. Precisam garantir a alimentação, o corpo aquecido, banho tomado e roupas limpas, sono no abrigamento, redução dos danos considerando a dependência de álcool e outras drogas, alívio da dor e sofrimento frente ao adoecimento, à solidão, ou às feridas quer visíveis ou invisíveis, sofridas ou causadas, que no momento do cuidado e atenção não foram julgadas pela equipe (Notas de campo).

Como apresentado pelos participantes deste estudo, uma potência no quotidiano da eCR é "a articulação em rede, porque não se faz promoção da saúde sozinho". A Figura 1 apresenta como se organiza a rede de atenção às pessoas em situação de rua, intra e intersetorial, e de solidariedade. Essa apresentação diagramada foi sugerida pelos participantes do estudo e validada em oficina, realizada em ambos os cenários, para retorno dos resultados às realidades pesquisadas.

\section{DISCUSSÃO}

O Consultório na Rua foi implementado como política pública de saúde para suprir historicamente a existência de uma lacuna

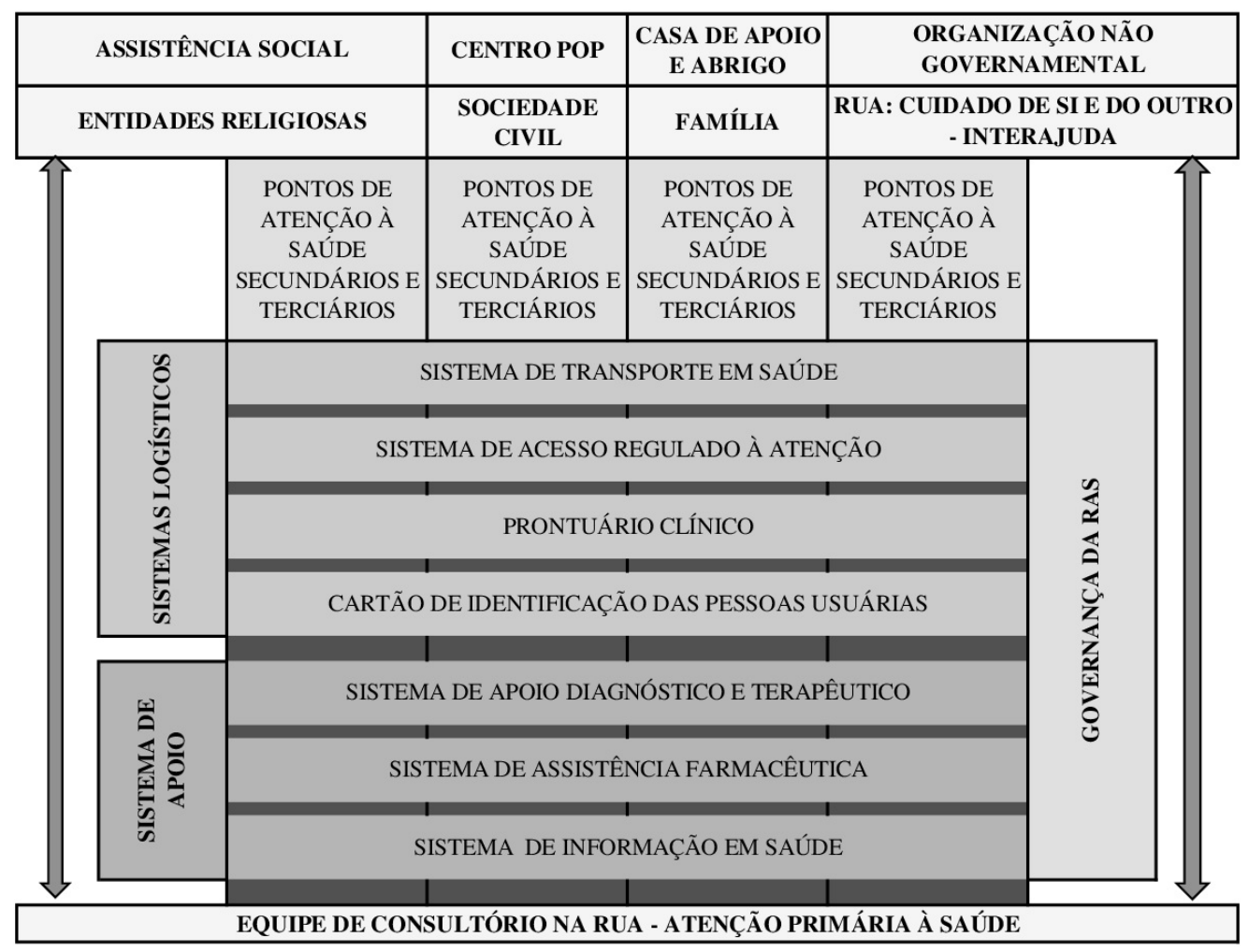

Figura 1. Consultório na Rua: a atenção à saúde de pessoas em situação de rua organizada em redes intrasetorial, intersetorial e de solidariedade.

Fonte: Adaptado de Mendes. ${ }^{27}$ 
assistencial das pessoas em situação de rua, privilegiando a abordagem dessas pessoas em seus contextos de vida e no cenário das ruas. O viver em condição de rua envolve situações e comportamentos de riscos, a distribuição desigual de bens sociais e o culmine da exclusão social.

A "Promoção da Saúde é um campo em construção e convive com uma pluralidade de concepções (modos de pensá-la) e de agir (intervenção)". 28:191 A promoção da saúde "como potência trata da passagem da passividade à atividade, da heteronomia à autonomia, da técnica à ética, da razão à emoção, do instituído ao instituinte". 28:193 Isso "não significa colocar cada uma dessas palavras-conceitos em posições antagônicas e ter de decidir por um ou outro polo. Ao contrário, significa o reconhecimento de inter-relações dinâmicas que são, ao mesmo tempo, objetivas e subjetivas, que há tendências ora para tencioná-las, ora para colocá-las lado a lado". ${ }^{28: 192-194}$

Destarte, as práticas em saúde, ao envolver dimensões sociais e filosóficas, simbólicas e culturais, devem considerar as subjetividades que perpassam o processo de ser saudável e adoecer. ${ }^{29}$ Considerando a articulação intrasetorial e colaboração intersetorial, no quotidiano da eCR, essas práticas perpassam a clínica ampliada em ações que extrapolam demandas da saúde e assentam no embate à exclusão social e à configuração da iniquidade na distribuição de recursos e de poder na sociedade.

Todavia, em um contexto de vulnerados, a crise atual se mostra societal por distanciar vidas, em que a própria vida é vista como ausente, negada pelo não querer ver ou reconhecer o vivido. ${ }^{30}$

Os resultados anuem, em ambos os casos estudados, que a demanda cotidiana está mais voltada para o tratamento do agravo do que para a promoção da saúde, porém a atenção às condições clínicas aproxima o ser profissional do ser vivendo na rua, estabelecendo-se o vínculo. Um estudo corrobora esses achados ao apresentar que a eCR identifica riscos e vulnerabilidades, e as pessoas em maior risco têm uma atenção mais premente voltadas para as demandas clínicas. Constituise a primeira forma de vínculo o atendimento clínico imediato, criando-se oportunidade para a longitudinalidade do cuidado, possibilitando planejar projetos terapêuticos, atenção psicossocial, incorporar ações educativas, de promoção da saúde, de apoio e de inclusão social. ${ }^{2}$

Um estudo realizado com pessoas em situação de rua leva à compreensão de que a lógica de funcionamento e organização das eCR está diretamente relacionada às condições imediatas de existência dessas pessoas. Portanto, para que essa população tenha saúde, é fundamental alterar as suas condições de existência. ${ }^{31}$ Para impactar sobre as condições de existência dessas pessoas, são necessárias ações intersetoriais para a promoção da saúde e de direitos sociais básicos, que ainda são negligenciados. A negligência coloca em xeque-mate o tempo futuro por formas formantes de vida ${ }^{32}$ ao desconsiderar parcela da população que convive com a insignificância pela exclusão.

Assim, se vive o presente na rua, o aqui e agora, no imediatismo, na resolutividade da necessidade demandada naquele instante de dor, da doença, da fome, do frio. A perspectiva hermenêutica está de acordo, metodologicamente, com o desenvolvimento das histórias de vida. Para a eCR, é necessário crescer junto com o que se brota. O olhar concreto se apega aos detalhes do quotidiano, às observações imediatas, a essa teatralidade da vida, nesse espaço, que é constituída de pequenas coisas, indiferentes como tais, mas que em seu conjunto ordenado, fazem sentido, ${ }^{32}$ a fim de possibilitar acesso à saúde e aos direitos humanos fundamentais às pessoas que vivem na rua.

A partir do depoimento dos profissionais da eCR, fica evidenciado a moradia e a fome, direitos humanos fundamentais. Neste cenário, revela-se a fome suprida por pessoas da comunidade, em rede de solidariedade, o que "tende a frisar o sentimento comunitário de pertencimento, ou seja, o processo de implicação por meio do qual cada um de nós existe em função do outro" 32:121. Deste modo, o alimento não nutre apenas o corpo, mas também nutre o estar junto, possibilitando uma solidariedade orgânica, do viver-comum Maffesoliano, que não é da ordem do instituído, do mecânico, mas sim pautada em uma ética da estética, do sentir junto, que se expressa pelo vínculo.

Ao considerar os elementos estruturais da dignidade humana resultante da convergência do ser humano com o estar humano, a ausência de dois dos direitos sociais, a alimentação e a habitação se tornam mais perceptíveis em relação às pessoas em situação de rua. O primeiro assegura a continuidade biológica o segundo, uma existência digna. ${ }^{33}$ Não ter acesso à moradia pode ter relação com fatores intrínsecos e extrínsecos, como distúrbio físico ou perda de trabalho. ${ }^{34}$

Apesar de as pessoas em situação de rua serem heterogêneas em relação ao perfil e às causas que as levaram a essa condição, há o que possuem em comum: "a pobreza extrema, os vínculos familiares interrompidos ou fragilizados e a inexistência de moradia convencional, seu teto são o sol e a lua, suas paredes são os papelões ou os viadutos e as pontes". ${ }^{35: 300}$. Desafios vitais que fazem parte dessa realidade na rua e dos determinantes do processo saúde-doença, e que demandam ações intersetoriais.

Assim, é preciso estar atento às 'coisas mesmas' e à sua lógica interna. É necessário compreender a cultura popular que mantém uma relação mágica com seu ambiente natural, com o mundo dos objetos, a interatividade que se estabelece com o mundo material constitutivo da vida quotidiana. ${ }^{32}$

Sopesando-se pessoas em situação de rua, um estudo realizado em Cheshire, condado localizado no nordeste da Inglaterra, aponta que as experiências que levam as pessoas a se tornarem sem-teto devem ser analisadas considerando-se as condições sociais em que a falta de moradia ocorreu, como a pobreza, a interrupção da escolaridade, o uso abusivo de drogas, a falta de apoio social e psicológico, o abuso físico, sexual e emocional, a negligência, os ambientes familiares disfuncionais e as estruturas familiares instáveis. Considera-se que tornar-se um sem-teto é um processo caracterizado por um progressivo declínio da resiliência criada por esses incidentes, levando-o a engajar-se em comportamento denominado de (des) 
adaptativo: abuso de substâncias, alcoolismo, autoflagelação e comportamentos disruptivos. ${ }^{36}$

Pode-se inferir a transfiguração da resiliência, da adaptação e da integração, visto que é preciso se adaptar e integrar-se ao mundo da rua, resistindo, insistindo, persistindo em viver, ou, melhor, sobreviver, visto que sempre é o evadir-se de riscos, enquanto que, em um paradoxo, é visto como um incômodo, expondo seu próprio limite.

Considerando a realidade dos sem-teto da cidade de Palermo, Sicília, Itália, um estudo realizado aponta que, apesar de o Artigo 32 da Constituição italiana declarar que para a proteção da saúde é fundamental direito humano para cada indivíduo e o interesse da comunidade, pouco foi feito em termos de planejamento de saúde para melhorar a qualidade de vida dos sem-teto e reduzir gastos em saúde. Seria aconselhável desenvolver uma estratégia preventiva de riscos e intervenções de saúde dirigidas a essas pessoas para melhorar suas condições de vida, além de permitir um acesso mais equitativo e acessível na saúde pública. ${ }^{34}$

A redução de danos foi ressaltada como uma prática cotidiana alternativa para a prevenção de riscos e de outros agravos, a educação em saúde e a atenção integral. O reconhecimento da singularidade dos usuários, pela equipe, permite traçar caminhos/ estratégias não excludentes, respeitando a liberdade e resiliência de cada um, para que sejam protagonistas de suas escolhas, mesmo em condições desfavoráveis na rua. ${ }^{18}$

Os participantes deste estudo anuem que só há atuação/ colaboração intersetorial e promoção da saúde das pessoas em situação de rua se houver uma gestão e cultura política que reconfigure novas formas de agir para minimização da exclusão social.

A política não está mais capacitada para enfrentar os desafios do momento, configurando-se como um objeto de desconfiança geral. O poder político é uma instância que determina a vida social, ou seja, limita-a, constrange-a e permite-Ihe existir. O governo tem o poder de decisão que compreende muitos âmbitos da sociedade. Porém, nota-se que o político só pode ser reconhecido enquanto tal se souber influenciar os sentimentos, os desejos e o imaginário coletivo. ${ }^{37}$

Para alcançar a promoção da saúde da população em situação de rua, é preciso reflexão sobre as políticas públicas e sua efetividade, a educação permanente dos profissionais, garantias de acesso à saúde e respeito às singularidades. ${ }^{38}$ Não tem como falar de direito à saúde e direitos humanos fundamentais à vida sem falar de justiça social. São necessárias políticas participativas e que o exercício da cidadania se constitua em espaço de participação popular para tomada de decisões e efetivação da saúde para todos.

Enfim, no quotidiano anunciado pelas eCR, para promover saúde, é preciso não apenas encaminhar, mas, sobretudo, caminhar junto às pessoas em situação de rua, tendo como fio condutor o vínculo, a escuta sensível, o toque, o diálogo, considerando seus limites e suas potências, lembrando que enquanto o poder é da ordem da dominação, a potência é da ordem da cooperação e da libertação. ${ }^{39}$

\section{CONCLUSÕES E IMPLICAÇÕES PARA A PRÁTICA}

Este estudo aponta que, para além da assistência às demandas de adoecimento/agravos, é preciso conceber e intervir em ações integradas e em rede para um enfoque social, político e técnico do processo saúde-doença que efetivamente promova saúde às pessoas em situação de rua, mesmo em condições de vida desfavoráveis. Estratégias intrasetoriais e intersetoriais devem ser estabelecidas para minimizar riscos e danos a que estão expostas as pessoas em situação de rua.

Assim, pensar a saúde como acesso à educação, trabalho, transporte, lazer, alimentação, dentre outros determinantes, implica a superação da exclusão social das pessoas em situação de rua e a defesa dos direitos fundamentais à vida.

A presença cotidiana da eCR no âmbito da rua traduz uma política que retoma a autonomia do controle dos determinantes sociais, estruturando-se em políticas intersetoriais visando impactar em condições mais humanas de vida de vulnerados. Enfim, entendese que é por esta via da promoção da saúde, no quotidiano, que residem as potencialidades de operacionalização de melhores condições de saúde para as pessoas em situação de rua.

\section{FINANCIAMENTO}

O presente trabalho foi realizado com apoio da Coordenação de Aperfeiçoamento de Pessoal de Nível Superior - Brasil (CAPES) - Código de Financiamento 001. A Bolsa do Programa Nacional de Pós-Doutorado (PNPD/CAPES-2018) foi concedida a Selma Maria da Fonseca Viegas. Foi também financiada por bolsa de iniciação científica pelo Edital 001/2018/PIBIC/CNPq/ UFSC, concedida a Lucas Andreolli Bernardo.

\section{CONTRIBUIÇÕES DOS AUTORES}

Desenho do estudo: Selma Maria da Fonseca Viegas.

Coleta ou produção dos dados: Selma Maria da Fonseca Viegas, Lucas Andreolli Bernardo.

Análise de dados: Selma Maria da Fonseca Viegas, Rosane Gonçalves Nitschke, Lucas Andreolli Bernardo.

Interpretação dos resultados: Selma Maria da Fonseca Viegas, Rosane Gonçalves Nitschke, Lucas Andreolli Bernardo, Adriana Dutra Tholl, Maria Aurora Rodriguez Borrego, Pablo Jesús López Soto, Daniela Priscila Oliveira do Vale Tafner.

Redação e revisão crítica do manuscrito: Selma Maria da Fonseca Viegas, Rosane Gonçalves Nitschke, Lucas Andreolli Bernardo, Adriana Dutra Tholl, Maria Aurora Rodriguez Borrego, Pablo Jesús López Soto, Daniela Priscila Oliveira do Vale Tafner.

Aprovação da versão final do artigo: Selma Maria da Fonseca Viegas, Rosane Gonçalves Nitschke, Lucas Andreolli Bernardo, Adriana Dutra Tholl, Maria Aurora Rodriguez Borrego, Pablo Jesús López Soto, Daniela Priscila Oliveira do Vale Tafner.

Responsabilidade por todos os aspectos do conteúdo e a integridade do artigo publicado: Selma Maria da Fonseca Viegas, Rosane Gonçalves Nitschke, Lucas Andreolli Bernardo, Adriana Dutra Tholl, Maria Aurora Rodriguez Borrego, Pablo Jesús López Soto, Daniela Priscila Oliveira do Vale Tafner. 


\section{EDITOR ASSOCIADO}

\author{
Aline Aparecida Monroe
}

\section{REFERÊNCIAS}

1. Carta de Ottawa. I Conferência Internacional sobre Promoção da Saúde. Ottawa, 21 de novembro de 1986. Genebra: OMS.

2. Engstrom EM, Teixeira MB. Equipe "Consultório na Rua" de Manguinhos, Rio de Janeiro, Brasil: práticas de cuidado e promoção da saúde em um território vulnerável. Ciênc saúde coletiva. 2016;21(6):1839-48. http://dx.doi.org/10.1590/1413-81232015216.0782016.

3. Kami MTM, Larocca LM, Chaves MMN, Piosiadlo LCM, Albuquerque GS. Saberes ideológicos e instrumentais no processo de trabalho no Consultório na Rua. Rev Esc Enferm USP. 2016 maio/jun;50(3):442-9. http://dx.doi.org/10.1590/S0080-623420160000400010. PMid:27556715.

4. Londero MFP, Ceccim RB, Bilibio LFS. Consultório de/na rua: desafio para um cuidado em verso na saúde. Interface Comunicacao Saude Educ. 2014 abr 30;18(49):251-60. http://dx.doi.org/10.1590/1807-57622013.0738.

5. Portaria $n^{\circ} 122$, de 25 de janeiro de 2011 (BR). Define as diretrizes de organização e funcionamento das Equipes de Consultório na Rua. Diário Oficial da União [periódico na internet], Brasília (DF), 2011 [citado 2020 jun 1]. Disponível em: http://189.28.128.100/dab/docs/legislacao/ portaria_122_25_01_2011.pdf

6. Ministério da Saúde (BR). Secretaria de Vigilância em Saúde. Secretaria de Atenção à Saúde. Política Nacional de Promoção da Saúde: PNPS: Anexo I da Portaria de Consolidação nº 2, de 28 de setembro de 2017, que consolida as normas sobre as políticas nacionais de saúde do SUS [Internet]. Brasília: Ministério da Saúde; 2018 [citado 2020 jun 1]. 40 p. Disponível em: http://bvsms.saude.gov.br/bvs/saudelegis/gm/2017/ prc0002_03_10_2017.html

7. Organização Pan-Americana da Saúde. Guia para Implementação das Prioridades Transversais na OPAS/OMS do Brasil: direitos humanos, equidade, gênero e etnicidade e raça. Brasilia: OPAS; 2018.

8. Aldridge RW, Story A, Hwang SW, Nordentoft M, Luchenski SA, Hartwell $\mathrm{G}$ et al. Morbidity and mortality in homeless individuals, prisoners, sex workers, and individuals with substance use disorders in high-income countries: a systematic review and meta-analysis. Lancet. 2018;391(10117):241-50. http://dx.doi.org/10.1016/S01406736(17)31869-X. PMid:29137869.

9. $\quad$ Liu CY, Chai SJ, Watt JP. Communicable disease among people experiencing homelessness in California. Epidemiol Infect. 2020;148:e85. http://dx.doi.org/10.1017/S0950268820000722. PMid:32223777.

10. Hino P, Santos JO, Rosa AS. People living on the street from the health point of view. Rev Bras Enferm. 2018;71(Suppl 1):684-92. http://dx.doi. org/10.1590/0034-7167-2017-0547. PMid:29562028.

11. Roche MA, Duffield C, Smith J, Kelly D, Cook R, Bichel-Findlay J et al. Nurse-led primary health care for homeless men: a multimethods descriptive study. Int Nurs Rev. 2018;65(3):392-9. http://dx.doi.org/10.1111/ inr.12419. PMid:29266302.

12. Paiva IKS, Lira CDG, Justino JMR, Miranda MGO, Saraiva AKM. Direito à saúde da população em situação de rua: reflexões sobre a problemática. Ciênc saúde coletiva. 2016 ago;21(8):2595-2606. http:// dx.doi.org/10.1590/1413-81232015218.06892015</jrn>.

13. Cantor JC, Chakravarty S, Nova J, Kelly T, Delia D, Tiderington E et al. Medicaid utilization and spending among homeless adults in New Jersey: implications for Medicaid-Funded tenancy suport services. Milbank Q. 2020;98(1):106-30. http://dx.doi.org/10.1111/1468-0009.12446. PMid:31967354.

14. Fazel S, Geddes JR, Kushel M. The health of homeless people in highincome countries: descriptive epidemiology, health consequences, and clinical and policy recommendations. Lancet. 2014 out;384(9953):152940. http://dx.doi.org/10.1016/S0140-6736(14)61132-6. PMid:25390578.

15. Zhang L, Norena M, Gadermann A, Hubley A, Russell L, Aubry T et al. Concurrent disorders and health care utilization among homeless and vulnerably housed persons in Canada. J Dual Diagn. 2018;14(1):21-31. $\mathrm{http}: / / \mathrm{dx}$.doi.org/10.1080/15504263.2017.1392055. PMid:29494795.

16. Sacamano P, Krawczyk N, Latkin C. Emergency department visits in a cohort of persons whit substance use: incorporating the role of social networks. Subst Use Misuse. 2018;53(13):2265-9. http://dx.doi.org/1 0.1080/10826084.2018.1461225. PMid:29671696.
17. Vijayaraghavan M, Apollonio DE. Engaging adults experiencing homelessness in smoking cessation through large-scale community sevice events. Health Promot Pract. 2019;20(3):325-7. http://dx.doi. org/10.1177/1524839919835280. PMid:30845844.

18. Simões TRBA, Couto MCV, Miranda L, Delgado PGG. Missão e efetividade dos Consultórios na Rua: uma experiência de produção de consenso. Saúde Debate. 2017;41(114):963-75. http://dx.doi. org/10.1590/0103-1104201711423.

19. Stergiopoulos V, Gozdzik A, Nisenbaum R, Durbin J, Hwang SW, O'Campo P et al. Bridging hospital and community care for homeless adults with mental health needs: outcomes of a brief interdisciplinary intervention. Can J Psychiatry. 2018;63(11):774-84. http://dx.doi. org/10.1177/0706743718772539. PMid:29716396.

20. Sofija E, Plugge M, Wiseman N, Harris N. 'This is the beginning of the new me': process evaluation of a group fitness intervention fo promote wellbeing in formerly homeless individuals. BMC Public Health. 2018;18(1):290. http://dx.doi.org/10.1186/s12889-018-5175-5. PMid:29482615.

21. Wijk LBV, Mângia EF. O cuidado a Pessoas em Situação de Rua pela Rede de Atenção Psicossocial da Sé. Saúde Debate. 2017;41(115):113042. http://dx.doi.org/10.1590/0103-1104201711511.

22. Nistchke RG, Tholl AD, Potrich T, Silva KM, Michelin SR, Laureano DD Contributions of Michel Maffesoli's Thinking to Research in Nursing and Health. Texto Contexto Enferm. 2017;26(4):e3230017. http://dx.doi. org/10.1590/0104-07072017003230017.

23. Yin RK. Estudo de caso: planejamento e métodos. $5^{\mathrm{a}}$ ed. Porto Alegre: Bookman; 2015.

24. Maffesoli M. O conhecimento comum: introdução à sociologia compreensiva. Porto Alegre: Sulina; 2010.

25. Maffesoli M. A terra fértil do cotidiano. Rev Famecos. 2008;15(36):5-9. http://dx.doi.org/10.15448/1980-3729.2008.36.4409.

26. Bardin L. Análise de conteúdo. São Paulo: Edições 70; 2011.

27. Mendes EV. As redes de atenção à saúde. Brasília: Organização PanAmericana da Saúde; 2011.

28. Mendes R, Fernandez JCA, Sacardo DP. Promoção da saúde e participação: abordagens e indagações. Saúde Debate. 2016;40(108):190-203. http:// dx.doi.org/10.1590/0103-1104-20161080016.

29. Silva AC, Ferreira J, Czeresnia D, Maciel EMGS, Oviedo RAM. Os sentidos da saúde e da doença. Ciênc. saúde coletiva. 2015;20(3):9578. http://dx.doi.org/10.1590/1413-81232015203.00212014.

30. Maffesoli M. O tempo retorna: formas elementares da pós-modernidade. Rio de Janeiro: Forense Universitária; 2012

31. De Tilio R, Oliveira JD. Cuidados e atenção em saúde da população em situação de rua. Psicol Estud. 2016;21(1):101-13. http://dx.doi. org/10.4025/psicolestud.v21i1.27142.

32. Maffesoli M. O ritmo da vida: variações sobre o imaginário moderno. Rio de Janeiro: Record; 2007.

33. Garcia E. Pessoas em situação de rua e direitos prestacionais. Revista Jus Navigandi [Internet]. 2014; [citado 2020 jun 1];19(4134). Disponível em: https://jus.com.br/artigos/32998

34. Alagna E, Santangelo OE, Raia DD, Gianfredi V, Provenzano S, Firenze A. Health status, diseases and vaccinations of the homeless in the city of Palermo, Italy. Ann Ig. 2019;31(1):21-34. http://dx.doi.org/10.7416/ ai.2019.2255. PMid:30554236.

35. Belizário DM. Os direitos fundamentais das pessoas em situação de rua: o Ministério Público como instituição garantidora desses direitos. De Jure (Durban). 2017;16(29):295-341. http://dx.doi.org/10.5935/18098487.20170013.

36. Mabhala MA, Yohannes A, Griffith M. Social conditions of becoming homelessness: qualitative analysis of life stories of homeless peoples. Int J Equity Health. 2017;16(1):150. http://dx.doi.org/10.1186/s12939017-0646-3. PMid:28830515.

37. Maffesoli M. A transfiguração do Pólíco e a tribalização do mundo. $3^{a}$ ed. Porto Alegre: Sulina; 2005.

38. da Silva FP, de Assis Siqueira Paiva F, Guedes CP, da Silva Frazão I, Vasconcelos SC, da Costa Lima MD. Nursing diagnoses of the homeless population in light of self-care theory. Arch Psychiatr Nurs. 2018;32(3):42531. http://dx.doi.org/10.1016/j.apnu.2017.12.009. PMid:29784225.

39. Maffesoli M. A ordem das coisas: pensar a pós-modernidade. Rio de Janeiro: Forense; 2016. 